\title{
Nondestructive Optical Visualization of Graphene Domains and Boundaries
}

Xingyi Wu, Guofang Zhong*, and John Robertson

X. Wu,Dr. G. Zhong, Prof. J. Robertson

Department of Engineering

University of Cambridge

Cambridge CB3 0FA, United Kingdom

E-mail: gz222@cam.ac.uk

keywords: nondestructive visualization, continuous graphene, domain boundary, ridge structure, dark field 
Large area, high quality, continuous monolayer graphene films are indispensable matrix for industrial manufacture of graphene-based high-end devices. Currently, graphene can be produced by mechanical exfoliation, ${ }^{[1]}$ epitaxial growth on $\mathrm{SiC},{ }^{[2,3]}$ chemical exfoliation, ${ }^{[4]}$ or chemical vapor deposition (CVD) on Ni, $\mathrm{Cu}$ and other substrates. ${ }^{[5,6]}$ Above all, Cu-catalyzed CVD (Cu-CVD) stands out as the most promising synthesis method for industrial production of graphene because it simultaneously fulfills industrial demands for high quality, monolayer uniformity, process scalability, and film continuity. ${ }^{[7]}$ Low lattice mismatch $(\sim 3-4 \%)$ between $\mathrm{Cu}$ and graphene renders $\mathrm{Cu}-\mathrm{CVD}$ graphene high quality comparable to mechanically exfoliated graphene. ${ }^{[8]}$ The $\mathrm{Cu}$-mediated self-limiting growth manner further facilitates precise control of monolayer uniformity. ${ }^{[6,9]}$ To date, roll-to-roll production of 100 meter-long graphene films has been demonstrated using the $\mathrm{Cu}-\mathrm{CVD} .^{[10]}$ Film completeness is conveniently guaranteed in $\mathrm{Cu}-\mathrm{CVD}$ by rationalized supply of carbon precursors. However, continuous graphene films grown by $\mathrm{Cu}-\mathrm{CVD}$ are always polycrystalline. ${ }^{[1,12]}$ The boundaries unavoidably emerge from the coalescence of nearby graphene domains. ${ }^{[6]}$ (Hereafter, "grain" and "domain" are used for polycrystalline $\mathrm{Cu}$ metal and graphene films, respectively.) The boundaries influence the graphene transporting properties and thereby alter the performances of graphene-based devices, ${ }^{[13-15]}$ which further causes detrimental inhomogeneity to the entire manufactured device arrays.

Recently, progresses have been made to drastically reduce the density of boundaries by increasing the domain sizes via modified Cu-CVD technologies. ${ }^{[16-19]}$ The largest domains by far have reached centimeter scale. ${ }^{[18]}$ On the other hand, the boundary associated adverse effects can be efficiently controlled by spatial visualization of the domains and boundaries. A potential visualization procedure can mark the boundary-infected devices after manufacture by mapping the domains and boundaries of the post-growth graphene films. The boundaryrelated products yield can then be evaluated and the quality reliability determined. In this 
sense, an effective visualization method is extraordinarily valuable for manufacture management of CVD graphene-related devices.

The graphene boundaries can be detected by atomic resolution microscopes such as TEM ${ }^{[11,12]}$ or STM ${ }^{[20,21]}$ and spectroscopic mappings like Raman. ${ }^{[22,23]}$ These methods are non-invasive with high accuracy but limited to much smaller scale than industry demanded. In recent years, scalable visualization approaches have been developed which include oxidizing the domain boundaries for optical microscopy detection ${ }^{[24]}$ and imaging the birefringence of nematic liquid crystal-coated graphene surfaces. ${ }^{[25,26]}$ The domain boundaries can also by mapped by tracking the aggregation patterns of gold nanoparticles deposited onto graphene surfaces. ${ }^{[27]}$ However, these methods are destructive to graphene since they have to either partially damage carbon bonds or induce contaminating coatings or transfer graphene onto secondary substrates. Therefore, a nondestructive and scalable visualization technique is urgently desired but still lacking.

In principle, if an ideal visualization method is to nondestructively map the graphene domains and boundaries, it should preferably detect certain intrinsic characteristics of graphene rather than any derivative physical or chemical effects induced by external interferences. Moreover, the potential 'intrinsic characteristics' should also be spatially specific to individual graphene domains so as to distinguish the locations of inter-connected domains. Based on this analysis and latest experimental findings, we have rationally designed a novel optical method, which, for the first time, can nondestructively visualize the domains and boundaries of large scale continuous graphene films grown on polycrystalline $\mathrm{Cu}$ foils $(\mathrm{Gr} / \mathrm{Cu})$.

Our design is inspired by recent discoveries of the dynamic pyramid formation on $\mathrm{Cu}$ surface covered by graphene domains before they merge into a continuous film during the $\mathrm{Cu}$ catalyzed graphene growth. ${ }^{[28-30]}$ The schematic drawing in Figure 1a illustrates this process. Basically, there is a pre-melted surface $\mathrm{Cu}$ layer at the typical graphene growth temperature of $\sim 1000^{\circ} \mathrm{C}$ (the melting temperature $T_{\mathrm{m}}$ of bulk $\mathrm{Cu}$ is about $1083^{\circ} \mathrm{C}$ in ambient conditions). The 
pre-melted $\mathrm{Cu}$ atoms are evaporated substantially and removed by the CVD carrier gas, ${ }^{[31]}$ which has a higher rate in graphene-free regions than in graphene-covered regions. ${ }^{[32,33]}$ Therefore, the $\mathrm{Cu}$ surface outside an enlarging graphene domain loses $\mathrm{Cu}$ atoms much faster than that below the domain. In the case of a hexagonal graphene domain, the $\mathrm{Cu}$ surface below the domain, which is originally flat, will evolve into a hexagonal pyramid structure due to the lateral evaporation (Figure 1a). ${ }^{[30]}$ Here we point out that, if viewed from the perpendicular direction, the hexagonal pyramid will appear as a six-ridge star. The ridges exactly follow the graphene domain diagonals since the pyramid structure inherits the geometric symmetry of the domain. Most remarkably, the ridge structure spatially tags the respective domain. If the ridge structures can be visualized in some route, the spatial distribution of graphene domains and domain boundaries can be straightforward determined through the spatial correspondence. Since the ridge structures are intrinsic features of the graphene- $\mathrm{Cu}$ interfacial morphology, the potential visualization process could be nondestructive.

Indeed, we find that the graphene- $\mathrm{Cu}$ interfacing ridge structures can be best imaged by a facilely modified optical microscope, which can be regarded as an enhanced dark field optical microscope (EDF-OM) due to its optical configuration. Figure 2a shows the setup of our EDF-OM which is developed from an upright OM. We only use an external illumination light source such as a desk lamp or an annular light instead of its original internal light source, as shown in Figure 2a and Figure S1, respectively (see Experimental Section for more details). The EDF-OM is then used to image discontinuous graphene samples which have been grown on commercial polycrystalline $\mathrm{Cu}$ foils via $\mathrm{CH}_{4}$ decomposition using an atmospheric pressure CVD recipe reported recently. ${ }^{[34]}$ Figure $2 b$ shows one of the EDF-OM images of isolated hexagonal graphene domains. A star structure is clearly revealed which consists of six ridges diverging from the domain center straightforward to the domain vertices. The entire structure is hence morphologically identical to the schematic in Figure 1. We will hereafter refer to the 
structures as the "ridge structures". In addition, feather-like features along some of the ridges are also observed, which make the whole domain much brighter than the surrounding $\mathrm{Cu}$ background. The star structures must arise from the intrinsic morphologies of the graphenecovered $\mathrm{Cu}$ surface because no such structures can be observed by EDF-OM on any graphene-free $\mathrm{Cu}$ surfaces.

Since the as-grown $\mathrm{Gr} / \mathrm{Cu}$ is invisible under bright-field $\mathrm{OM}(\mathrm{BF}-\mathrm{OM})$, a mild post-oxidation treatment is needed to enable BF-OM observation of the isolated domain. ${ }^{[35]}$ Figure $2 \mathrm{c}$ displays a BF-OM image of the same domain obtained after mild oxidation. It only shows a hexagonal domain with a homogenous color, providing no clues for the ridge structure. The enormous difference between the EDF OM and BF-OM observation is further demonstrated by a real time recording in Supporting Video 1, which demonstrates the evolution from Figure $2 \mathrm{c}$ to Figure $2 \mathrm{~b}$ under constant external light intensity and varying internal light intensity (between max and zero). Such a difference originates from their different optical configurations. The external light source of the EDF-OM provides large-angle oblique incident light $\left(\sim 50^{\circ}\right.$ relative to the sample plane, see Figure $\left.2 \mathrm{a}\right)$, while the internal BF light source gives almost vertical incident light with a much smaller incident angle and stronger intensity. Under EDF-OM, most of the oblique incident light from the external light source will be specularly reflected away from the objective by any smooth surfaces. However, as the star-like ridges experience large curvature changes between two adjacent pyramid faces, these ridges could scatter enough light into the objective which thereby visualizes the ridges themselves. On the contrary, under BF-OM, most of the vertical internal incident light will be reflected back into the objective, which results in no obvious contrast between the ridges and the pyramid faces. We find that a pristine DF-OM using the hollow cone-shaped internal light can also visualize the ridge structures, but the brightness is too weak and the contrast too poor, which thereby might be easily ignored by other researchers. ${ }^{[36]}$ This is probably because of the weak intensity and small incident angle of the internal DF light. We regard our setup shown in 
Figure $2 \mathrm{a}$ as the EDF-OM also because of the external source-enhanced incident angle and light intensity.

Similar to the BF-OM, the low magnification SEM image of the same domain in Figure $2 \mathrm{~d}$ barely shows any ridge-like features. This agrees with a large number of SEM studies reporting homogenous color inside $\mathrm{Cu}$-catalyzed graphene domains. ${ }^{[37]}$ However, close observations under high SEM magnifications along the domain diagonals strongly indicate the existence of these star-like ridges, which appear slightly brighter than the surrounding background, as shown in Figure 2e and Figure S2a-f. This thereby supports the EDF-OM discovery of the star-like ridge structure. It should be noted that this is the first time that such a ridge structure is formally manifested by SEM. However, although SEM can capture an intrinsically identical ridge structure as the EDF-OM, it only generates much weaker contrast. Thereby the EDF-OM is much more competent than the high magnification SEM in imaging the graphene ridge structures for visualization of large scale graphene domains and boundaries.

In addition to the well-defined star-like six-ridge structure, we have further discovered, for the first time, a plethora of novel graphene structures which do not always exhibit six ridges, as summarized in Figure 3a-f and Figure S3. These structures differ from each other. For instance, the structures in Figure 3a-e have visible ridges while that in Figure $3 \mathrm{f}$ has no ridges. Furthermore, it can be seen from the EDF-OM image of multiple types of ridge structures (Figure 3g) and the corresponding electron backscattering diffraction (EBSD) map of the underlying $\mathrm{Cu}$ grains (Figure 3h) that different graphene ridge structures are formed on different $\mathrm{Cu}$ grains while those on the same $\mathrm{Cu}$ grain are of exactly the same type. Complete EBSD analysis of the $\mathrm{Cu}$ grains for Figure 3a-f has further confirmed this correlation (Miller indexes are marked inside Figure $3 \mathrm{a}-\mathrm{f}$ and also illustrated by their outline colors). These strongly suggest that the underlying $\mathrm{Cu}$ crystallographic orientations govern the formation of the ridge structures. In particular, a graphene domain grown over multiple $\mathrm{Cu}$ grains, as seen 
in Figure 3c, has mixed ridge structures in accordance with the crystallographic orientations of the respective $\mathrm{Cu}$ grains. It should be noted that this is the first time the diverse $\mathrm{Cu}$ crystallographic orientation-dependent ridge structures are discovered, although there has been intense research on polycrystalline $\mathrm{Cu}$-catalyzed graphene growth and its characterization. ${ }^{[38]}$ This is most likely because their morphological differences are clearly observable under our novel EDF-OM but hardly noticeable under the commonly used BF-OM and SEM. Although the three dimensional laser microscopy has found a graphene ridge structure close to that in Figure $3 \mathrm{a},{ }^{[29]}$ it cannot detect all of the other types. The various ridge structures thereby raise new phenomenon for fundamental graphene research beyond the current scope.

As the visibility of the ridge structure is determined by the curvature change between the adjacent pyramid faces, the smaller is the dihedral angle $\beta$ (Figure $1 \mathrm{~b}$ ), the higher will be the ridge visibility. For simplicity, considering the case of the hexagonal $\mathrm{Cu}$ pyramid illustrated in Figure 1a, $\beta$ can be calculated as:

$$
\sin \frac{\beta}{2}=\sqrt{\frac{1+\left(\frac{h}{a}\right)^{2}}{1+\frac{4}{3} \cdot\left(\frac{h}{a}\right)^{2}}} \approx 1-\frac{1}{6} \cdot\left(\frac{h}{a}\right)^{2}, h \ll a
$$

where $h$ is the height of the pyramid and $a$ is the lateral length of the hexagonal graphene domain. Assuming both the lateral growth rate of graphene, $\gamma_{g r}$, and the evaporation rate of uncovered $\mathrm{Cu}, \gamma_{\mathrm{Cu}}$, are constant, Equation 1 can be further approximated by:

$$
\sin \frac{\beta}{2} \approx 1-\frac{1}{6} \cdot\left(\frac{\gamma_{C u}}{\gamma_{g r}}\right)^{2}
$$

Here, $\gamma_{C u}$ depends on the CVD processing temperature $T$, the standard vapor pressure of $\mathrm{Cu}$ $P_{\text {cu }}$ and total ambient pressure $P,{ }^{[39]}$ while $\gamma_{g r}$ can be taken proportional to the partial pressure of hydrocarbon gas $P_{\mathrm{C}}$ in a first-order reaction:

$$
\begin{gathered}
\gamma_{C u}=\frac{P_{C u}-P}{\sqrt{2 \pi M R T}}, \\
\gamma_{g r}=k \cdot P_{C},
\end{gathered}
$$


where $M$ is the molecular weight of $\mathrm{Cu}, R$ is the universal gas constant, and $k$ is the first-order graphene growth rate constant. Equations 2-4 relate the ridge visibility with the CVD graphene growth kinetics. The conditions using high processing temperature $\left(\sim 1000{ }^{\circ} \mathrm{C}\right)$ and low hydrocarbon concentration for synthesis of high quality $\mathrm{Cu}-\mathrm{CVD}$ graphene favour the formation of observable ridge structures. ${ }^{[40,41]}$ Considering that atmospheric pressure is used throughout our CVD processes, graphene grown by the most commonly used low pressure CVD ( 1Torr) should have more pronounced ridges due to faster $\mathrm{Cu}$ evaporation. ${ }^{[41]}$ In addition, it should be noted that the visibility of the ridge structures is also influenced by the crystallographic orientations of the $\mathrm{Cu}$ grains as previoysly shown in Figure 3. During $\mathrm{Cu}$ CVD processes, high temperature annealing is used to promote formation of large $\mathrm{Cu}$ grains with low-energy crystallographic surfaces, mostly (110), (100) and (111) (The absence of these exact low-index surfaces from our EBSD results is probably because of the deformation of the $\mathrm{Cu}$ foils during sample handling). It has been well established that the the thermal stability of the low-index fcc metal surfaces follows their in-plane packing density. Generally the relativly open (110) and (100) surfaces start to disorder and pre-melt at temperature below $T_{\mathrm{m}}$ while the most densely packed (111) surface keeps in a crystalline state up to $T_{\mathrm{m}}$ and shows weak superheating effects. ${ }^{[42,43]}$ This can well explain our observations in Figure 3. Since a lower stability results in a higher evaporation rate of $\mathrm{Cu}$, the visibility of graphene ridge structures under EDF-OM varies on different $\mathrm{Cu}$ crystallographic surfaces. As the (111) surface is of the highest stability, the ridge structures can hardly form on (111)-vicinal $\mathrm{Cu}$ surfaces under our CVD conditions, therefore the as-grown graphene domains on (111)vicinal $\mathrm{Cu}$ surfaces are barely visible under EDF-OM (see Figure 3f). However, most of the graphene domains grown on other $\mathrm{Cu}$ grains are more or less visible because of their relatively lower surface stability (see Figure 3a-e). As for the additional features making parts of the graphene domains brighter, particularly noticeable in Figure $3 \mathrm{~b}$ and $3 \mathrm{e}$, their formation probably lies in the reconstruction of $\mathrm{Cu}$ surface under the template of graphene domains 
during both the isothermal growth and cooling process. ${ }^{[30,44]}$ This is essential since the graphene-covered $\mathrm{Cu}$ pyramid faces are usually in higher energy states compared to the lowenergy surfaces of the graphene-free $\mathrm{Cu}$ grains formed during annealing. The reconstruction, particularly during the cooing process, will transform the atomic flat pre-melted $\mathrm{Cu}$ into 'stepwise rough' facets, ${ }^{[30]}$ some of which might be rough enough to be visualized by our EDF-OM. The reconstruction is also dependent on the crystallographic properties of $\mathrm{Cu}$ grains. ${ }^{[30,44]}$ It should also be noted that if the graphene growth rate is so high as to rapidly form continuous graphene layer, or the growth temperature is too low which suppresses the evaporation of $\mathrm{Cu}$, the ridge structures can hardly form on $\mathrm{Cu}$ and thereby the graphene domains would be barely visible under the EDF-OM.

By far, we have confirmed that the star-like ridge structures arise from the intrinsic morphologies of graphene-Cu interfacing and can be clearly imaged by EDF-OM. We now demonstrate the non-destructive visualization of continuous $\mathrm{Gr} / \mathrm{Cu}$ and its application in outlining the domain boundaries and estimating the domain density and the average size. Figure 4a-c show three typical images, taken by EDF-OM, BF-OM and SEM, respectively, of a continuous $\mathrm{Gr} / \mathrm{Cu}$. The EDF-OM image in Figure 4a clearly demonstrates multiple sets of star-like ridge structures. In contrast, the BF-OM and SEM images in Figure $4 \mathrm{~b}$ and $4 \mathrm{c}$ merely show the $\mathrm{Cu}$ surface morphology with a uniform contrast, which agrees with previous investigations. $^{[6,36,37]}$ As each set of star-like ridge structure spatially fingerprints a graphene domain, we can use the ridge intersection to mark the nucleation center of each graphene domain and separate the neighourboring domains by outlining each set of the ridge structure according to the length of the diverging ridges. Figure 4d shows the same EDF-OM image as Figure 4a with individual domains approximately outlined by the yellow polygons based on their corresponding ridges. The graphene nucleation centers (ridge intersections) are generally deviated away from the geometrical centers of the domains, which is due to the competitive growth of neighboring domains. ${ }^{[30]}$ The domain density in Figure $4 \mathrm{~d}$ is counted to be $\sim 5.5$ 
$\mathrm{mm}^{-2}$ and thus the average domain size is $\sim 0.18 \mathrm{~mm}^{-2}$. Figure $4 \mathrm{e}$ and $4 \mathrm{f}$ further illustrate the EDF-OM images of the continuous graphene grown on other $\mathrm{Cu}$ grains under the same CVD conditions with their domains outlined by the yellow polygons. It should be noted that our optical visualization approach works only under the EDF-OM mode. This is further demonstrated in Supporting Video 2 recording the evolution of the OM image from Figure 4b to Figure 4a with constant external light intensity and varying internal light intensity.

Since the graphene domain density gradually saturates as coverage increases during Cu-CVD growth, ${ }^{[45]}$ we substantiate the reliability of our visualization method by comparing the domain density and size distribution visualized at full coverage to those counted at partial coverage. Figure $4 \mathrm{~g}$ plots the graphene domain density against the corresponding coverage. The coverage is measured from both the EDF-OM and BF-OM images, but the $\mathrm{Gr} / \mathrm{Cu}$ sample is mildly oxidized for BF-OM observations. The domain density up to $\sim 30 \%$ coverage can be accurately counted from the BF-OM image since most domains are spatially isolated. However, the BF-OM method begins to cause noticeable errors after $\sim 60 \%$ coverage and completely fails at $>80 \%$ coverage as an increasing number of domains are becoming interconnected. In contrast, the EDF-OM approach can figure out the domain density from zero up to full coverage. We can see from Figure $4 \mathrm{~g}$ that the visualized domain density at full coverage agrees well with the counted domain density at lower coverage $(\sim 30 \%$ and $\sim 60 \%)$ with limited discrepancy $(\sim 14 \%)$. Figure 4 h shows that the domain size distribution at full coverage (determined by EDF-OM) is also in consistence with that at partial coverage (determined by both EDF-OM and BF-OM). Figure 4i shows that there is no significant discrimination for the domain densities of discontinuous graphene counted from crystallographically different $\mathrm{Cu}$ grains. This is consistent with previous studies using similar growth temperature ${ }^{[46]}$. These observations justify that our EDF-OM visualization method is effective and reliable for continuous graphene films although the ridge structure of the domains grown on (111)-vicinal $\mathrm{Cu}$ grains are less visible than the rest. 
We highlight that our visualization method has significant advantages over the up-to-date methods. First of all, it is nondestructive since we directly observe the as-grown $\mathrm{Gr} / \mathrm{Cu}$ samples by an optical microscope with no need for oxidation, assistive coatings and transfer. Moreover, it is scalable, cost-saving, and rapid because an optical microscope works in ambient conditions by frame-to-frame scan.

In conclusion, we have developed a novel EDF-OM method for visualizing the domains and domain boundaries of continuous $\mathrm{Gr} / \mathrm{Cu}$ in a non-destructive, scalable, cost-saving and rapid manner. It is based on a variety of novel star-like ridge structures which are in one-to-one spatial correspondence to the $\mathrm{Cu}$-catalyzed graphene domains. The ridge structures arise from the evaporation rate differences between the graphene covered and uncovered $\mathrm{Cu}$ areas. Reconstruction of $\mathrm{Cu}$ under the template of graphene domains also affects the visibility of the domain by the EDF-OM. The star-like ridge structures vary on different $\mathrm{Cu}$ grains because both the formation of the ridge structures and the reconstruction of $\mathrm{Cu}$ surfaces are highly dependent on the $\mathrm{Cu}$ crystallographic orientations. This research opens a new route towards probing the graphene domain boundaries for assessing and controlling the boundaryassociated adverse effects on industry-scale manufacture of graphene-based devices. Meanwhile, the multi-types of $\mathrm{Cu}$ grain-dependent ridge structures enrich current understanding of the complexity of graphene-Cu interfacing dynamics.

\section{Experimental Section}

Graphene synthesis: Graphene synthesis on $\mathrm{Cu}$ foils is carried out by atmospheric pressure CVD. ${ }^{[34]}$ Electropolished $\mathrm{Cu}$ foils (Alfa Aesar, $25 \mu \mathrm{m}$ thickness, 99.8\% purity) are used as catalysts. Ramping and annealing is carried out in Ar atmosphere $(490 \mathrm{sccm})$ at $1030{ }^{\circ} \mathrm{C}$ (total pressure $\sim 1$ bar, heating rate $\sim 40{ }^{\circ} \mathrm{C} / \mathrm{min}$ ). In a benchmark one-step CVD process, $\mathrm{CH}_{4}$ $(\sim 0.12 \mathrm{sccm})$ and $\mathrm{H}_{2}(10 \mathrm{sccm})$ are then added to the Ar gas followed by cooling in $\mathrm{Ar}$ and $\mathrm{H}_{2}$. 
It takes $\sim 95$ min to grow continuous monolayer graphene films. Isolated graphene domains are obtained by terminating the $\mathrm{CH}_{4}$ supply before the domains merge.

Characterization: The $\mathrm{Gr} / \mathrm{Cu}$ samples are characterized by optical microscope (Nikon ECLIPSE LV150N) in both the EDF and the BF modes, SEM (Zeiss SIGMA VP) and EBSD (Oxford NordlysMax EBSD detector mounted on Philips XL30 sFEG SEM). For EDF-OM observations, an external illumination light source such as a desk lamp or an annular light is used instead of its original internal light source. To record the supporting videos, the external light source is kept on constant intensity while the internal light is manually decreased from a high intensity to zero and then back to the initial intensity. For BF-OM observations of isolated graphene domains, the pristine $\mathrm{Gr} / \mathrm{Cu}$ sample is mildly oxidized in air at $200{ }^{\circ} \mathrm{C}$ for 1 $\min .^{[35]}$

\section{Supporting Information}

\section{Acknowledgements}

The authors acknowledge funding from XXX. They also thank Girish Roughbour for help with three dimensional schematic drawings. X.W. acknowledges funding from St. John's College, University of Cambridge, and a scholarship Cambridge Overseas Trust. 


\section{References}

[1] K. S. Novoselov, A. K. Geim, S. V. Morozov, D. Jiang, Y. Zhang, S. V. Dubonos, I. V. Grigorieva, A. A. Firsov, Science 2004, 306, 666.

[2] C. Berger, Z. Song, T. Li, X. Li, A. Y. Ogbazghi, R. Feng, Z. Dai, A. N. Marchenkov, E. H. Conrad, P. N. First, W. A. de Heer, J. Phys. Chem. B 2004, 108, 19912.

[3] C. Berger, Z. Song, X. Li, X. Wu, N. Brown, C. Naud, D. Mayou, T. Li, J. Hass, A. N. Marchenkov, E. H. Conrad, P. N. First, W. A. de Heer, Science 2006, 312, 1191.

[4] S. Stankovich, D. A. Dikin, G. H. B. Dommett, K. M. Kohlhaas, E. J. Zimney, E. A. Stach, R. D. Piner, S. T. Nguyen, R. S. Ruoff, Nature 2006, 442, 282.

[5] Q. Yu, J. Lian, S. Siriponglert, H. Li, Y. P. Chen, S.-S. Pei, Appl. Phys. Lett. 2008, 93, 113103.

[6] X. Li, W. Cai, J. An, S. Kim, J. Nah, D. Yang, R. Piner, A. Velamakanni, I. Jung, E. Tutuc, S. K. Banerjee, L. Colombo, R. S. Ruoff, Science 2009, 324, 1312.

[7] S. Hofmann, P. Braeuninger-Weimer, R. S. Weatherup, J. Phys. Chem. Lett. 2015, 6, 2714.

[8] A. S. Mayorov, R. V. Gorbachev, S. V. Morozov, L. Britnell, R. Jalil, L. A. Ponomarenko, P. Blake, K. S. Novoselov, K. Watanabe, T. Taniguchi, A. K. Geim, Nano Lett. 2011, 11, 2396.

[9] X. Li, W. Cai, L. Colombo, R. S. Ruoff, Nano Lett. 2009, 9, 4268.

[10] T. Kobayashi, M. Bando, N. Kimura, K. Shimizu, K. Kadono, N. Umezu, K. Miyahara, S. Hayazaki, S. Nagai, Y. Mizuguchi, Y. Murakami, D. Hobara, Appl. Phys. Lett. 2013, 102, 023112.

[11] P. Y. Huang, C. S. Ruiz-Vargas, A. M. van der Zande, W. S. Whitney, M. P. Levendorf, J. W. Kevek, S. Garg, J. S. Alden, C. J. Hustedt, Y. Zhu, J. Park, P. L. McEuen, D. A. Muller, Nature 2011, 469, 389.

[12] K. Kim, Z. Lee, W. Regan, C. Kisielowski, M. F. Crommie, A. Zettl, ACS Nano 2011, 5, 2142.

[13] O. V. Yazyev, S. G. Louie, Nat. Mater. 2010, 9, 806.

[14] O. V. Yazyev, S. G. Louie, Phys. Rev. B 2010, 81, DOI 10.1103/PhysRevB.81.195420.

[15] L. A. Jauregui, H. Cao, W. Wu, Q. Yu, Y. P. Chen, Solid State Commun. 2011, 151, 1100.

[16] Z. Yan, J. Lin, Z. Peng, Z. Sun, Y. Zhu, L. Li, C. Xiang, E. L. Samuel, C. Kittrell, J. M. Tour, ACS Nano 2012, 6, 9110.

[17] H. Zhou, W. J. Yu, L. Liu, R. Cheng, Y. Chen, X. Huang, Y. Liu, Y. Wang, Y. Huang, X. Duan, Nat. Commun. 2013, 4, DOI 10.1038/ncomms3096.

[18] Y. Hao, M. S. Bharathi, L. Wang, Y. Liu, H. Chen, S. Nie, X. Wang, H. Chou, C. Tan, B. Fallahazad, H. Ramanarayan, C. W. Magnuson, E. Tutuc, B. I. Yakobson, K. F. McCarty, Y.-W. Zhang, P. Kim, J. Hone, L. Colombo, R. S. Ruoff, Science 2013, 342, 720.

[19] A. Mohsin, L. Liu, P. Liu, W. Deng, I. N. Ivanov, G. Li, O. E. Dyck, G. Duscher, J. R. Dunlap, K. Xiao, G. Gu, ACS Nano 2013, 7, 8924.

[20] K. W. Clark, X.-G. Zhang, I. V. Vlassiouk, G. He, R. M. Feenstra, A.-P. Li, ACS Nano 2013, 7, 7956.

[21] L. Gao, J. R. Guest, N. P. Guisinger, Nano Lett. 2010, 10, 3512.

[22] Q. Yu, L. A. Jauregui, W. Wu, R. Colby, J. Tian, Z. Su, H. Cao, Z. Liu, D. Pandey, D. Wei, T. F. Chung, P. Peng, N. P. Guisinger, E. A. Stach, J. Bao, S.-S. Pei, Y. P. Chen, Nat. Mater. 2011, 10, 443.

[23] S. Wang, S. Suzuki, H. Hibino, Nanoscale 2014, 6, 13838.

[24] D. L. Duong, G. H. Han, S. M. Lee, F. Gunes, E. S. Kim, S. T. Kim, H. Kim, Q. H. Ta, K. P. So, S. J. Yoon, S. J. Chae, Y. W. Jo, M. H. Park, S. H. Chae, S. C. Lim, J. Y. Choi, Y. H. Lee, Nature 2012, 490, 235.

[25] D. W. Kim, Y. H. Kim, H. S. Jeong, H.-T. Jung, Nat. Nanotechnol. 2012, 7, 29. 
[26] J.-H. Son, S.-J. Baeck, M.-H. Park, J.-B. Lee, C.-W. Yang, J.-K. Song, W.-C. Zin, J.-H. Ahn, Nat. Commun. 2014, 5, 3484.

[27] S. U. Yu, B. Park, Y. Cho, S. Hyun, J. K. Kim, K. S. Kim, ACS Nano 2014, 8, 8662.

[28] J. M. Wofford, S. Nie, K. F. McCarty, N. C. Bartelt, O. D. Dubon, Nano Lett. 2010, 10, 4890.

[29] S. Zhu, Q. Li, Q. Chen, W. Liu, X. Li, J. Zhang, Q. Wang, X. Wang, H. Liu, RSC Adv. 2014, 4, 32941.

[30] Z.-J. Wang, G. Weinberg, Q. Zhang, T. Lunkenbein, A. Klein-Hoffmann, M. Kurnatowska, M. Plodinec, Q. Li, L. Chi, R. Schloegl, M.-G. Willinger, ACS Nano 2015, 9, 1506.

[31] S. Chen, H. Ji, H. Chou, Q. Li, H. Li, J. W. Suk, R. Piner, L. Liao, W. Cai, R. S. Ruoff, Adv. Mater. 2013, 25, 2062.

[32] J. S. Bunch, S. S. Verbridge, J. S. Alden, A. M. van der Zande, J. M. Parpia, H. G. Craighead, P. L. McEuen, Nano Lett. 2008, 8, 2458.

[33] N. S. Mueller, A. J. Morfa, D. Abou-Ras, V. Oddone, T. Ciuk, M. Giersig, Carbon 2014, $78,347$.

[34] X. Wu, G. Zhong, L. D’Arsié, H. Sugime, S. Esconjauregui, A. W. Robertson, J. Robertson, Sci. Rep. 2016, 6, 21152.

[35] C. Jia, J. Jiang, L. Gan, X. Guo, Sci. Rep. 2012, 2, DOI 10.1038/srep00707.

[36] X. H. Kong, H. X. Ji, R. D. Piner, H. F. Li, C. W. Magnuson, C. Tan, A. Ismach, H. Chou, R. S. Ruoff, Appl. Phys. Lett. 2013, 103, 043119.

[37] J. Xie, J. P. Spallas, Tech. Rep. Agil. Technol. 2012.

[38] J. D. Wood, S. W. Schmucker, A. S. Lyons, E. Pop, J. W. Lyding, Nano Lett. 2011, 11, 4547.

[39] J. Safarian, T. A. Engh, Metall. Mater. Trans. A 2013, 44, 747.

[40] P. R. Kidambi, C. Ducati, B. Dlubak, D. Gardiner, R. S. Weatherup, M.-B. Martin, P. Seneor, H. Coles, S. Hofmann, J. Phys. Chem. C 2012, 116, 22492.

[41] R. Muñoz, C. Gómez-Aleixandre, Chem. Vap. Depos. 2013, 19, 297.

[42] B. Chatterjee, Nature 1978, 275, 203.

[43] H. Häkkinen, M. Manninen, Phys. Rev. B 1992, 46, 1725.

[44] K. Hayashi, S. Sato, N. Yokoyama, Nanotechnology 2013, 24, 025603.

[45] T. Terasawa, K. Saiki, Nat. Commun. 2015, 6, DOI 10.1038/ncomms7834.

[46] I. Vlassiouk, S. Smirnov, M. Regmi, S. P. Surwade, N. Srivastava, R. Feenstra, G. Eres, C. Parish, N. Lavrik, P. Datskos, S. Dai, P. Fulvio, J. Phys. Chem. C 2013, 117, 18919. 
(a)

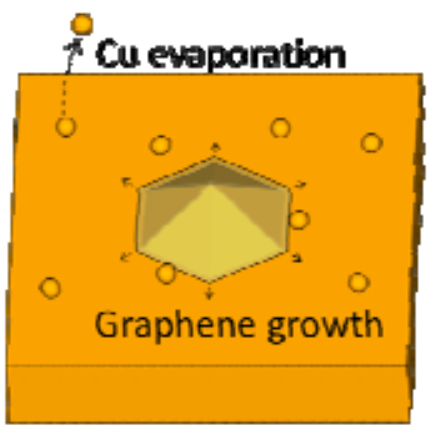

(b)
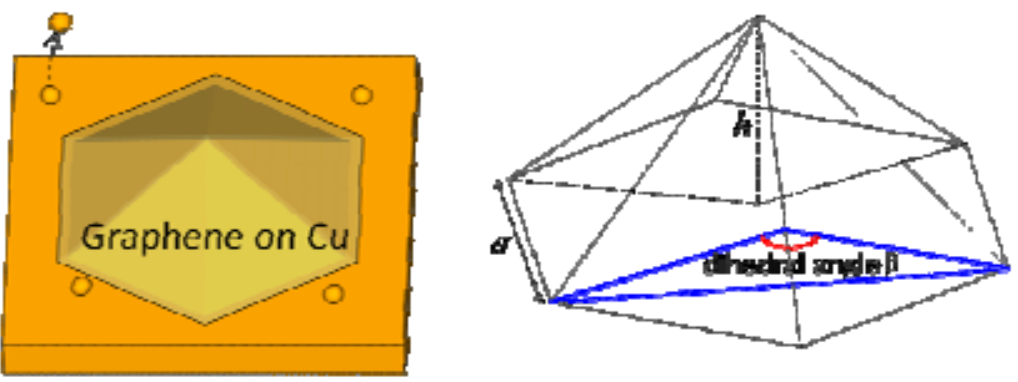

Cinonth thine

Figure 1. (a) Schematic diagram of the formation of a graphene domain-templated $\mathrm{Cu}$ pyramid due to the different evaporation rates of $\mathrm{Cu}$ between the graphene covered and uncovered areas. (b) Sketch of the hexagonal pyramid for calculation of the dihedral angle. 

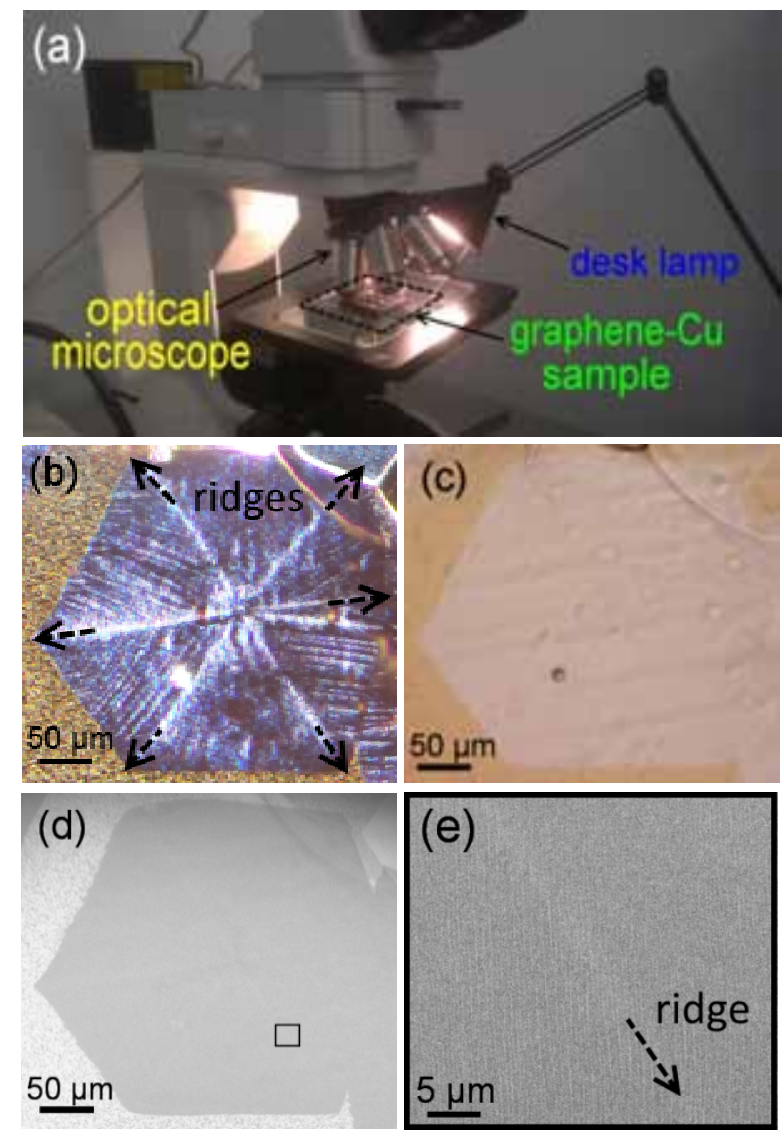

Figure 2. (a) Setup of the EDF-OM, which uses an external illumination light source such as a desk lamp instead of its original internal light source. (b) EDF-OM, (c) BF-OM and (d) SEM images of the same isolated hexagonal graphene domain grown on $\mathrm{Cu}$. The $\mathrm{Gr} / \mathrm{Cu}$ sample has been mildly oxidized for BF-OM observation. The six-ridge star structure is observable only by the EDF-OM. (e) High magnification SEM image of a midpoint along one of the domain diagonals marked by the black open box in (d). The black dashed arrow marks the location and orientation of the ridge. 

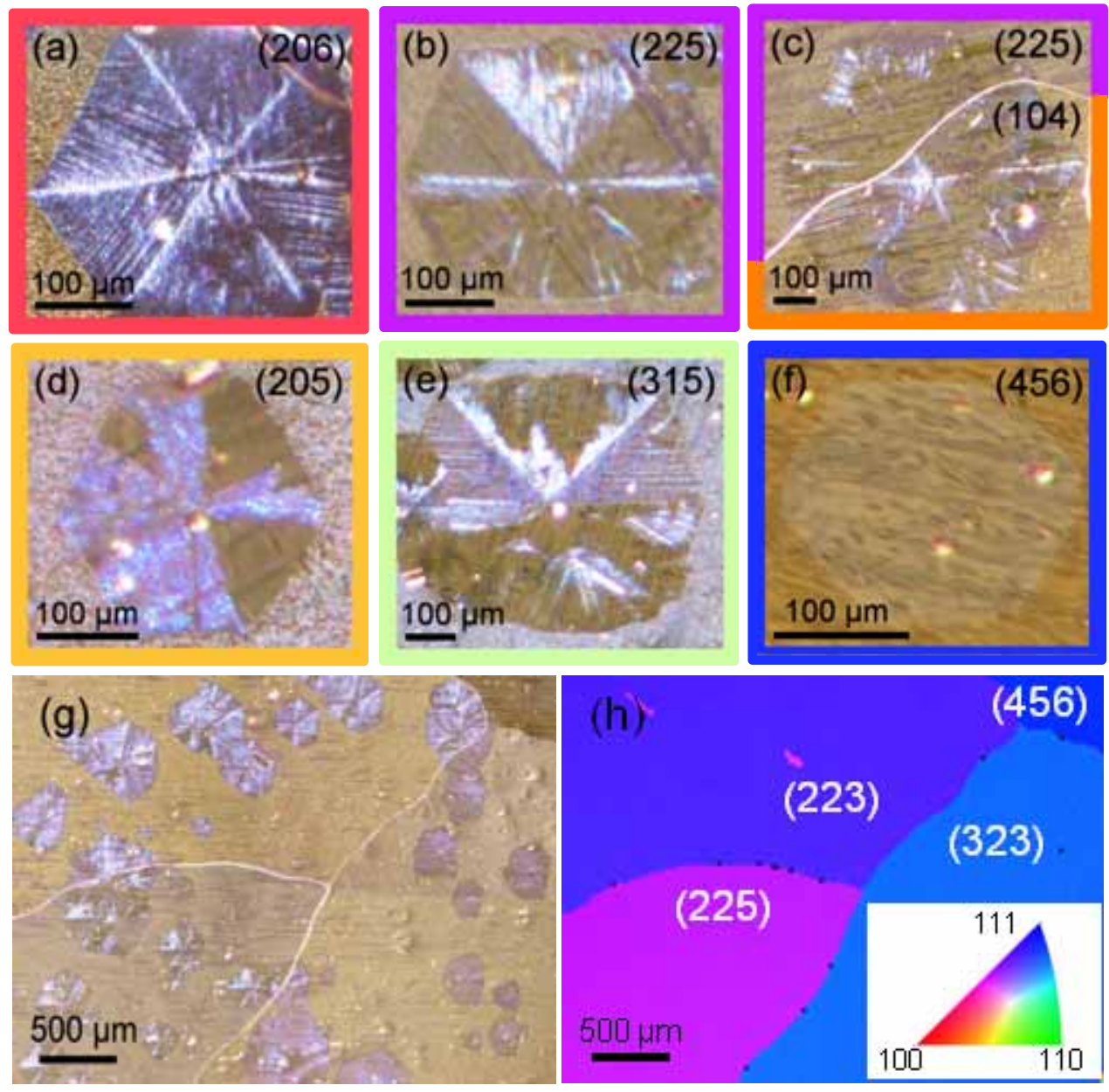

Figure 3. (a)-(f) EDF-OM images of multi-types of ridge structures with EBSD-determined Miller indexes of the underlying $\mathrm{Cu}$ grains marked inside each panel. The outline colors are set equal to the corresponding EBSD mapping colors of the respective $\mathrm{Cu}$ surfaces. The mixcolored outline of (c) indicates the presence of two different $\mathrm{Cu}$ grains below one single crystalline graphene domain. (g) EDF-OM image of the graphene domains with various ridge structures grown over a polycrystalline $\mathrm{Cu}$ region. (h) Surface normal-projected inverse pole figure EBSD map of the same region as in $(\mathrm{g})$ with the color key inset. The sample is tilted at $70^{\circ}$ for EBSD scanning and the resulted Miller indexes are marked inside each $\mathrm{Cu}$ grain. 

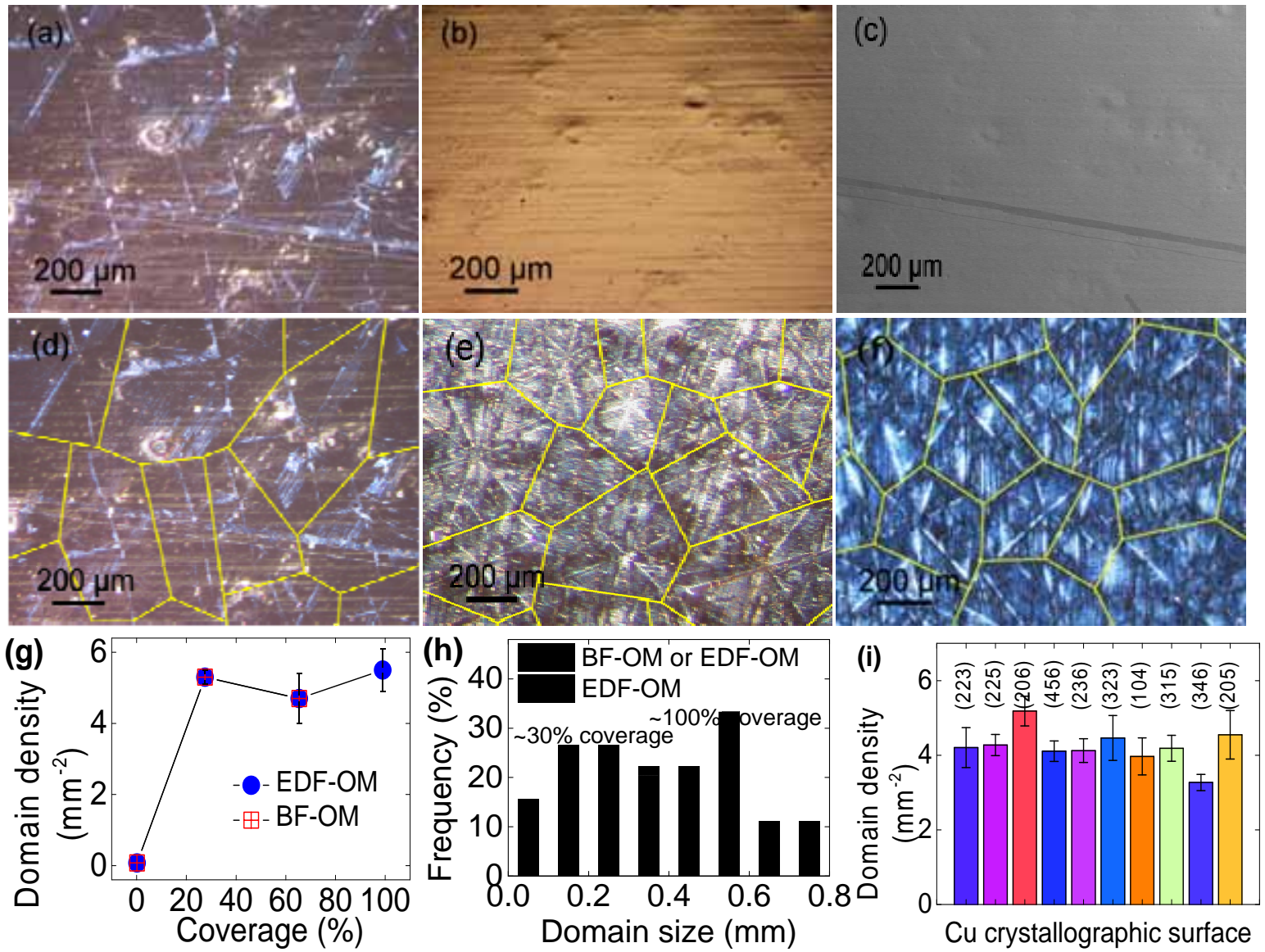

Figure 4. (a) EDF-OM, (b) BF-OM and (c) SEM images of a typical continuous $\mathrm{Gr} / \mathrm{Cu}$. Only the EDF-OM can visualize the star-like ridge structures that are spatially corresponding to the graphene domains. (d-f) Outlining the graphene domains of continuous graphene films by the solid yellow polygons according to the fingerprint star-like ridge structures visualized by the EDF-OM. The solid polygons represent the approximate domain boundaries. The graphene films in (d-f) are obtained from crystallographically different $\mathrm{Cu}$ grains under the same CVD conditions. (g) Graphene domain density against coverage. CVD graphene with different coverage is grown by varying the carbon supply time. (f) Distributions of graphene domain sizes at $\sim 100 \%$ coverage (blue histograms) and $\sim 30 \%$ coverage (red histograms), determined by the EDF-OM and the BF-OM, respectively. (i) Graphene domain density against $\mathrm{Cu}$ crystallographic orientations, which is counted from discontinuous $\mathrm{Gr} / \mathrm{Cu}$. The column colors are set equal to the EBSD mapping colors of the respective $\mathrm{Cu}$ surfaces. 